\title{
O custo da precariedade: o colapso da saúde dos editores é também o colapso da revista
}

\author{
Vinícius Medina Kern \\ Doutor; Universidade Federal de Santa Catarina, Florianópolis, SC, Brasil; \\ v.m.kern@ufsc.br; ORCID: https://orcid.org/0000-0001-9240-304X \\ Mauricio Uriona-Maldonado \\ Doutor; Universidade Federal de Santa Catarina, Florianópolis, SC, Brasil; \\ m.uriona@ufsc.br; ORCID: https://orcid.org/0000-0002-1174-4828
}

\begin{abstract}
Resumo: As revistas científicas brasileiras de ciência da informação têm dotação de recursos precária, com casos de descontinuidade na publicação, interrupção de submissões e grandes atrasos na avaliação de manuscritos, com possível relação com a capacidade e a saúde de seus editores. Qual o efeito do aumento da carga de trabalho no estresse dos editores? Neste artigo, simulamos quatro cenários do estresse de editores dessas revistas baseados em dados de quantidades de submissões, avaliações e decisões editoriais. Usamos dinâmica de sistemas para modelar estoques e fluxos de artigos e verificar quatro cenários do estresse de editores de revistas por 72 meses, com distintas cargas de submissão. Em dois cenários, com cargas de submissão moderadas, os editores mantêm o estresse e o tempo total de avaliação em níveis aceitáveis, inclusive aumentando a produtividade sob pequena sobrecarga, em acordo com o que diz a literatura. Nos dois cenários com maior sobrecarga de submissões, a quantidade de horas trabalhadas por mês cresce muito, bem como o tempo total de avaliação e o estresse acumulado. Um sistema de publicação que não provê recursos suficientes para o trabalho editorial de alta qualidade em tempo adequado corrói a saúde dos editores e favorece interrupções de submissões, atrasos e até o colapso das revistas.
\end{abstract}

Palavras-chave: Periódicos científicos; Acesso aberto; Dinâmica de sistemas; Publicação científica; Produtivis mo acadêmico; Revisão por pares

\section{Introdução}

O colapso da revista científica Ciência da Informação, já no século XXI, foi marcante para a área, num momento em que se destacava como principal periódico brasileiro da área. A interrupção por vários anos sem publicar edições foi registrada em apenas um artigo na literatura (BLATTMANN; SANTOS, 2014) e foi situação precursora do estado atual das revistas científicas brasileiras 
de ciência da informação: há casos de descontinuidade, interrupção (suspensão, moratória) de submissões e grandes atrasos na avaliação de manuscritos.

Há indícios de que nossas revistas mais prestigiadas são as mais vulneráveis ao colapso. Rosa et al. (2020) calcularam o tempo médio de aprovação dos artigos em revistas brasileiras de ciência da informação por estrato Qualis 2013-2016 da Coordenação de Aperfeiçoamento de Pessoal de Nível Superior (CAPES, 2018). Encontraram que os estratos mais elevados tomam mais tempo entre submissão e aprovação. Os tempos medianos são de 35 dias (estrato C, um periódico na amostra), 85 dias (estrato B5, 3 periódicos), 69 dias (estrato B2, 2 periódicos), 124 dias (estrato B1, 4 periódicos), 141 dias (estrato A2, 3 periódicos) e 187 dias (estrato A1, 3 periódicos).

No debate após a apresentação do artigo precursor desta pesquisa, no VI EBBC - Encontro Brasileiro de Bibliometria e Cientometria (KERN; URIONAMALDONADO, 2018b), um editor de periódico mensal da área da Saúde relatou sua rotina intensa, que implicava na impossibilidade de gozar férias, para evitar atraso irrecuperável no trabalho editorial. Esse testemunho de editor é compatível com a tendência de sobrecarga de cientistas observada por Barnett, Mewburn e Schroter (2019), ao verificarem que autores e revisores submetem manuscritos e pareceres de revisão fora de horários usuais de trabalho: "trabalhar das 9 às 5 não é uma maneira de ganhar a vida acadêmica" (p. 1).

Anteriormente (KERN; URIONA-MALDONADO, 2018), mostramos dados históricos das quantidades de submissões, avaliações por pares e rejeições preliminares (desk rejects). Esses dados históricos foram insumos para um estudo de cenários com quantidades distintas de submissões, com ou sem possíveis intervenções para aliviar a carga de trabalho editorial: moratória de submissões e aumento agudo de rejeições preliminares.

O comentário do editor sem férias inspirou a pesquisa relatada neste artigo. Consideramos que a saúde dos editores é um recurso não contabilizado que mantém em funcionamento as revistas brasileiras líderes na Ciência da Informação em acesso aberto e sem taxas, uma vez que a dotação de recursos para o funcionamento dessas revistas costuma ser precário, tipicamente 
envolvendo algumas horas no plano de trabalho do(s) editor(es), computador(es) e bolsista(s) que auxiliam nos aspectos burocráticos do trabalho dos editores.

Relatos pessoais de outros editores e alguns estudos publicados registram a incerteza ou a insuficiência de recursos alocados à edição de revistas em acesso aberto sem taxas (MUELLER, 1999, CORREA; MIRANDA, 2011, SHINTAKU et al., 2018). Considerando essa tensão entre a dotação precária de recursos e a necessidade de manter o trabalho editorial, esta pesquisa trata de prospectar cenários do funcionamento dessas revistas sob diversas cargas de submissão de artigos e seus efeitos na entrega de artigos e no estresse dos editores.

Este artigo amplia uma comunicação oral (KERN; URIONAMALDONADO, 2020) apresentada sumariamente no VII Encontro Brasileiro de Bibliometria e Cientometria (EBBC). As próximas seções apresentam os procedimentos metodológicos, os resultados, que incluem um modelo do processo editorial em dinâmica de sistemas e a descrição de quatro cenários de funcionamento sob diferentes cargas de submissão de manuscritos, bem como a discussão do significado e implicação desses resultados.

\section{Procedimentos metodológicos}

Esta seção delineia a abordagem da dinâmica de sistemas, principal método da pesquisa. A seguir, apresenta os materiais e as etapas para a simulação de cenários com dinâmica de sistemas.

\subsection{Método: dinâmica de sistemas}

A dinâmica de sistemas (STERMAN, 2000) é uma técnica de simulação criada em meados do século XX e vem sendo usada para modelar estoques e fluxos em sistemas complexos e descrever cenários alternativos de funcionamento, tanto para antever desdobramentos futuros quanto para estimar como se chegou a um estado atual. "The limits to growth" (MEADOWS et al., 1972) é um exemplo do primeiro caso: ao compor cenários futuros com macrovariáveis como população, produção industrial, poluição e recursos não-renováveis, os autores alcançaram 
fama mundial quando as décadas seguintes confirmaram as projeções (MEADOWS et al., 2004), conforme a Figura 1. Já a recomposição de um cenário atual cujas causas e desdobramentos não estão claros é o que fizeram Wu, Leung e Leung (2020) a partir de dados históricos pontuais, para estimar a dinâmica inicial do desenvolvimento da pandemia de COVID-19.

Figura 1 - Simulação do comportamento de macrovariáveis no estudo "The limits to growth" original (1972) com ajuste no período 1970-2000, no estudo reeditado (2004)

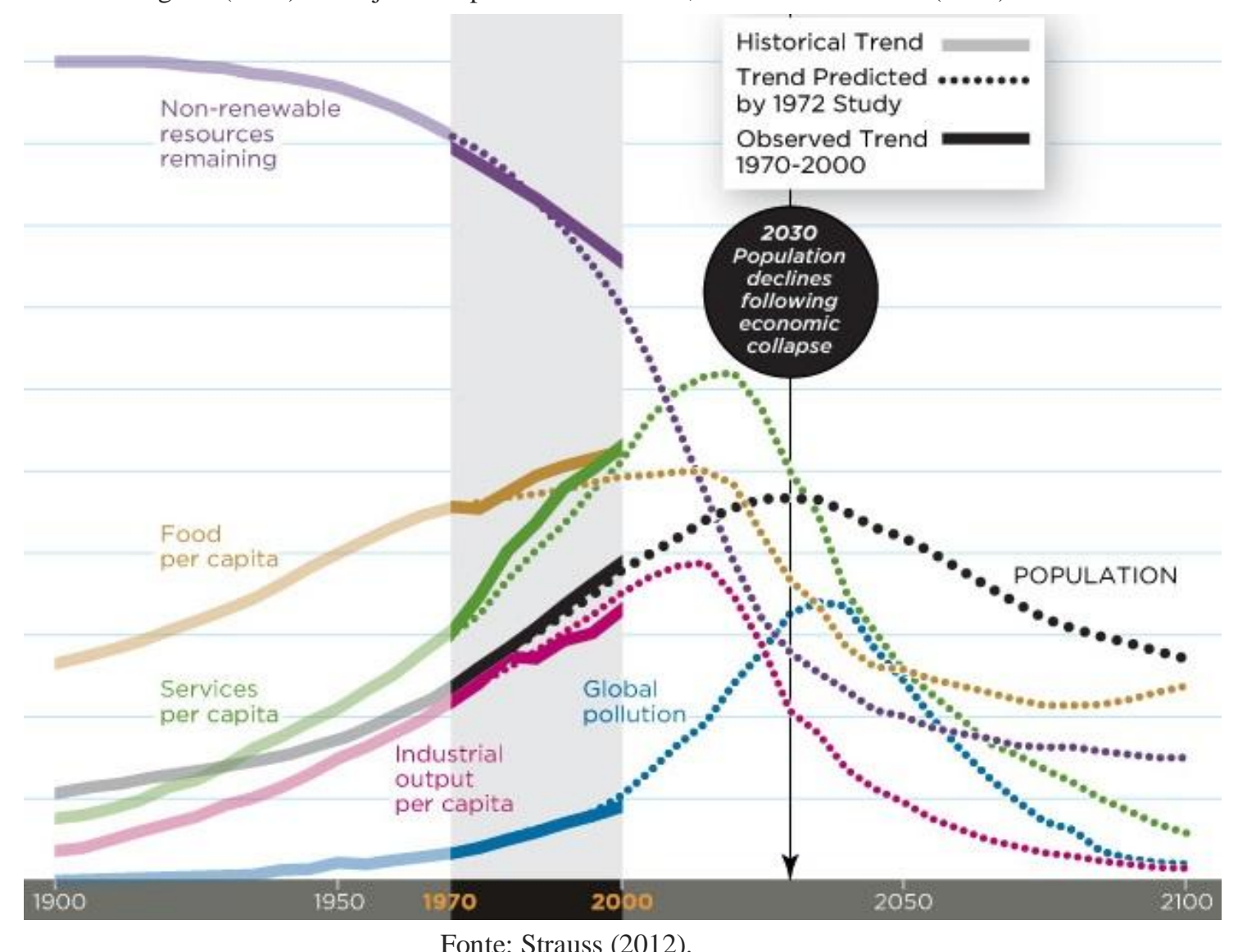

Fonte: Strauss (2012).

A dinâmica de sistemas é usada quando o problema em estudo possui propriedades dinâmicas, isto é: (i) quando a interação entre as principais variáveis acontece ao longo do tempo; (ii) quando acumulações e fluxos de informação, energia ou matéria são relevantes para a compreensão do problema; (iii) quando é importante salientar as relações de retroalimentação entre as variáveis; e (iv) quando as relações causais entre as variáveis relevantes não são completamente compreendidas (URIONA-MALDONADO; GROBBELAAR, 2019). 
Neste artigo, modelamos a interação entre a carga de trabalho de editores científicos de revistas brasileiras (representada pelo estoque ou acumulação de artigos que se encontram em alguma etapa do processo editorial, bem como o fluxo desses artigos ao longo das diferentes etapas do processo) com a geração de estresse provocada pelo gerenciamento dessa carga, por parte dos editores, e a sua dissipação no tempo (efeitos pouco estudados e compreendidos, desde o ponto de vista da ciência da informação).

Os modelos de simulação de dinâmica de sistemas, para tanto, utilizam uma linguagem de representação que salienta os estoques, fluxos e efeitos da retroalimentação ao longo do tempo (STERMAN, 2000). Na Figura 2, o item A mostra os principais elementos de forma genérica, representando os estoques por retângulos, os fluxos por válvulas e os ciclos de retroalimentação por setas azuis sequenciais. $\mathrm{O}$ item $\mathrm{B}$, na Figura 2, mostra um exemplo estilizado para a temática deste estudo, considerando o estoque como a quantidade de artigos em avaliação a qualquer momento do tempo, os fluxos de entrada (manuscritos, artigos iniciando avaliação) e saída (artigos avaliados), bem como o ciclo de retroalimentação que aumenta ou diminui a velocidade das avaliações a partir da comparação ou distância entre os artigos em avaliação (estoque) e a meta definida pela equipe editorial ${ }^{1}$. 
(B)

Figura 2 - Metamodelo (A) e modelo de fluxo de artigos em dinâmica de sistemas
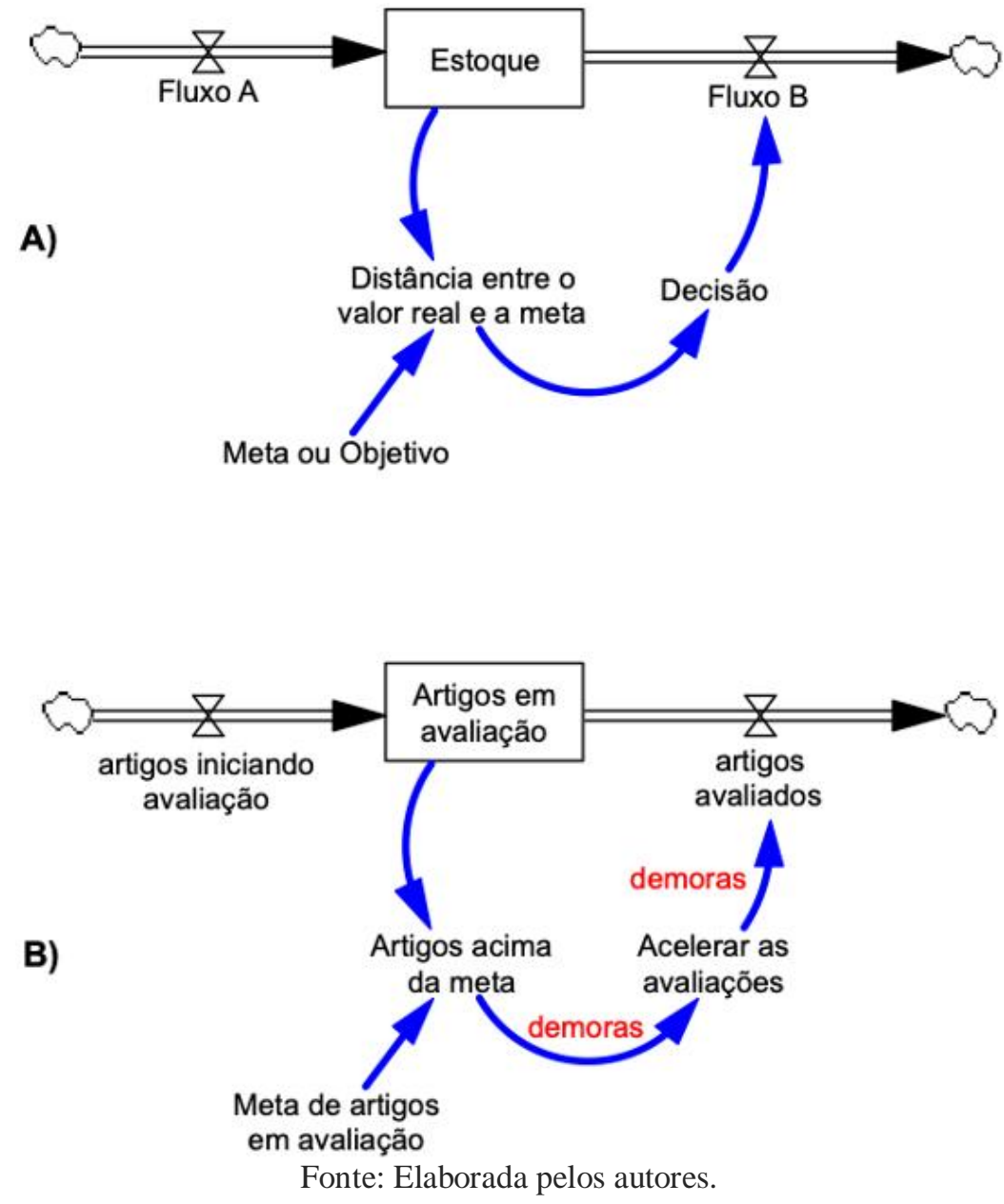

O ciclo de retroalimentação da Figura 2B é conhecido como ciclo de balanceamento (URIONA-MALDONADO; GROBBELAAR, 2019), porque gera uma resposta - nesse caso, a aceleração das avaliações - para tentar diminuir o número de artigos que estão acima da meta. Em outras palavras, procura ajustar a quantidade de artigos em avaliação com a meta definida. $\mathrm{Na}$ prática, haverá demoras tanto para decidir sobre a aceleração ou não das avaliações quanto para efetivamente observar um incremento ou diminuição da vazão de artigos avaliados, que podem depender de fatores humanos ou tecnológicos, o que faz com que a gestão desse sistema seja mais complexa do que inicialmente aparenta.

Por esse motivo, os modelos de simulação de dinâmica de sistemas são resolvidos utilizando equações diferenciais (equações que expressam taxas de variações), que representam o nível do estoque como função das entradas e 
saídas (fluxos) e são resolvidos por software especializado. No caso do exemplo da Figura 2A, a equação diferencial para o estoque é dada pela equação 1:

$$
\frac{d}{d t} \text { Estoque }=F_{A}-F_{B}
$$

Onde FA: Somatório dos Fluxos de entrada;

FB: Somatório dos Fluxos de saída.

Ou seja, num momento específico do tempo, a taxa de variação do estoque é dada pela diferença entre os fluxos que entram e saem do estoque. Note-se que, no exemplo da Figura 2A, o fluxo FB também depende de outras variáveis. A qualidade da simulação em dinâmica de sistemas depende da qualidade do modelo e da fundamentação em valores iniciais verossímeis, como descreve a próxima seção.

\subsection{Materiais e etapas de modelagem}

Para a execução das simulações, utilizamos o software de dinâmica de sistemas Stella Architect 1.7.1. É um software que permite criar diagramas de estoques e fluxos e simular seu comportamento ao longo do tempo (ISEE SYSTEMS, 2021).

O levantamento de dados atualizou as estatísticas de um estudo anterior (KERN; URIONA-MALDONADO, 2018), publicadas por algumas das revistas brasileiras primárias ativas de Ciência da Informação nos estratos Qualis (20132016) A1, A2 e B1. Constatamos nessa atualização (Figura 3) que, dentre oito revistas que ainda divulgam esses dados, a média mensal de submissões foi de 10,2 em 2018 e 11,2 em 2019. As maiores médias mensais recentes foram as da Perspectivas em Ciência da Informação, de 22,7 submissões em 2018, e 19,5 em 2019, da RECIIS - Revista Eletrônica de Comunicação, Informação e Inovação em Saúde, seguida de perto pela Ciência da Informação. 


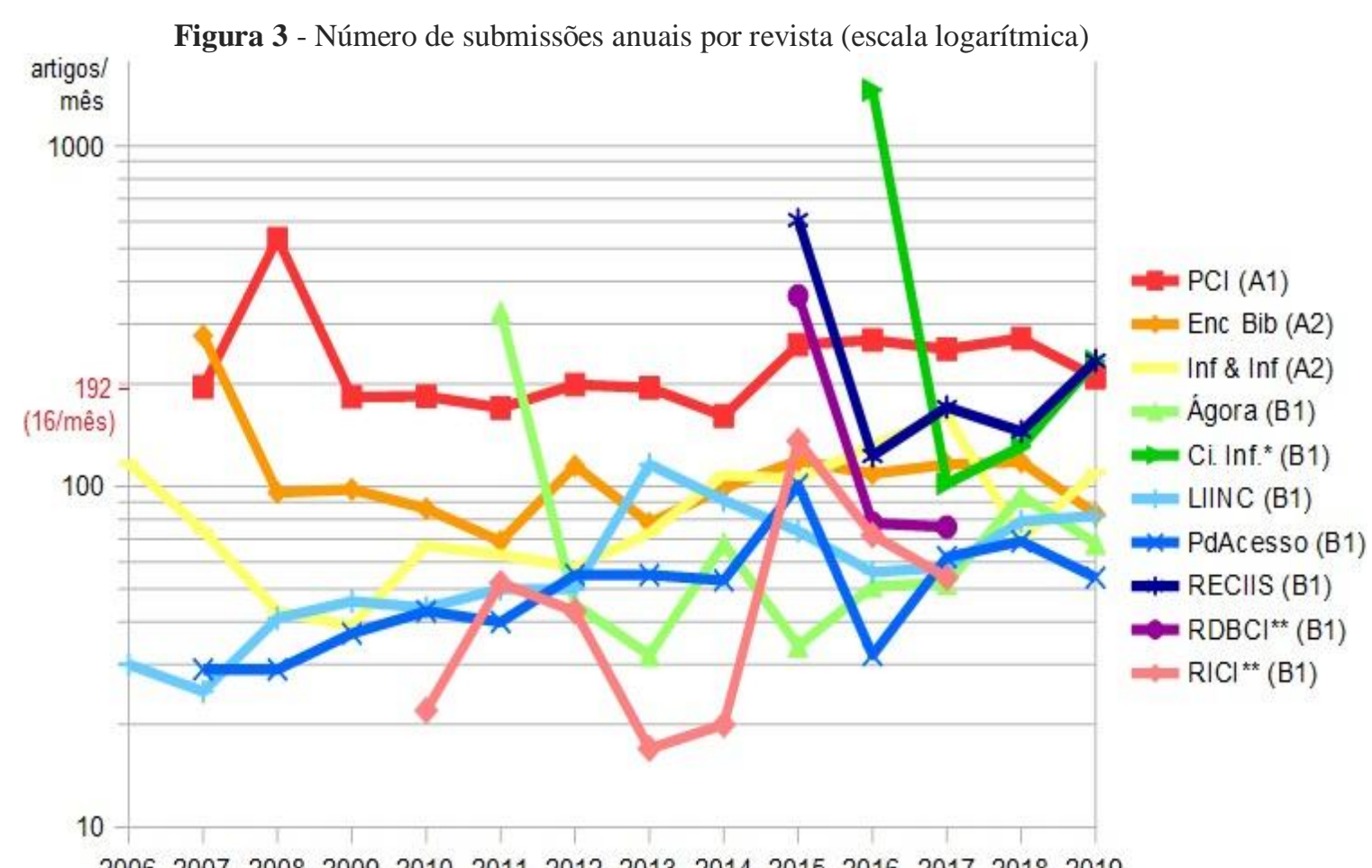

20062007200820092010201120122013201420152016201720182019

LEGENDA:

* Dados inexistentes na pesquisa anterior (KERN; URIONA-MALDONADO, 2018).

** Dados coletados em 12/2017 e indisponíveis em 02/2020.

Fonte: Elaborada pelos autores a partir de estatísticas das revistas.

Também atualizamos as estatísticas das revistas sobre quantidades de artigos enviados para revisão por pares, aceitos e rejeitados, bem como os tempos de avaliação e publicação. Simulamos os cenários com valores semelhantes aos do estudo anterior (KERN; URIONA-MALDONADO, 2018), conforme a Figura 4.

Figura 4 - Cenários do processo editorial: distintas condições de funcionamento

\begin{tabular}{|l|l|}
\hline Cenário (cor) & Características \\
\hline BAU (lilás) & BAU (business-as-usual): fluxo contínuo mensal de 16 submissões com 6 rejeições na triagem. \\
\hline A1 (vermelho) & Idem BAU, com aumento de 10\% de novas submissões (total 18) no mês 12. \\
\hline A2 (verde) & Idem BAU, com aumento de 30\% de novas submissões (total 21) no mês 12. \\
\hline A3 (azul) & Idem BAU, com aumento de 50\% de novas submissões (total 24) no mês 12. \\
\hline
\end{tabular}
Fonte: Elaborada pelos autores.

A modelagem é atividade artesanal, iterativa e dependente de perícia. É uma abstração informada por dados empíricos ou por estimativas. Os modelos 
de dinâmica de sistemas são criados em quatro estágios (RANDERS, 1980): conceituação, formulação, testes e implementação.

Conceituar é definir um propósito para o modelo, seus limites, variáveis e o comportamento dessas variáveis. Também é criar um diagrama usando construtos básicos da dinâmica de sistemas - laços de causa e efeito, estoques e fluxos (a próxima seção, Resultados, mostra diagramas de estoques e fluxos).

Formular é atribuir equações matemáticas aos elementos do diagrama, bem como estabelecer os valores dos parâmetros. Testar é executar a simulação, descrevendo o comportamento das variáveis no tempo e sua sensibilidade a perturbações conforme as equações e parâmetros.

Implementar é consolidar as equações e os valores de parâmetros e registrar a resposta do modelo a diferentes políticas ou cenários. A partir desses procedimentos, a próxima seção apresenta o modelo construído e quatro cenários de funcionamento para um periódico brasileiro de Ciência da Informação (hipotético, não específico) sob condições diversas de submissão de manuscritos, de acordo com a Figura 4. A próxima seção apresenta os modelos e cenários construídos.

\section{Resultados: modelos e simulação de cenários}

O modelo de estoques e fluxos da Figura 5 mostra quatro estoques (caixas rotuladas) que recebem e transmitem fluxos (ícones de registros sobre setas vazadas pretas) de artigos. Esses estoques e fluxos são afetados por variáveis (rótulos que recebem e emitem setas azuis) que podem, por sua vez, ser afetadas por outras variáveis, quantidades de estoques ou taxas de fluxos. Em particular (em vermelho na Figura 5), as variáveis "efeito do stress [dos editores] na produtividade" e "efeito do stress [dos editores] na qualidade dos artigos aceitos" são calculadas a partir de um modelo de dinâmica de sistemas adaptado para o caso dos editores a partir de um modelo de geração e dissipação de estresse de equipes de enfermagem por Sant'ana, Uriona-Maldonado e Gontijo (2019), conforme a Figura 6 


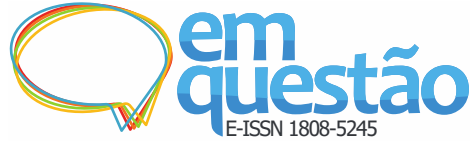

Figura 5 - Modelo de estoques e fluxos do processo editorial

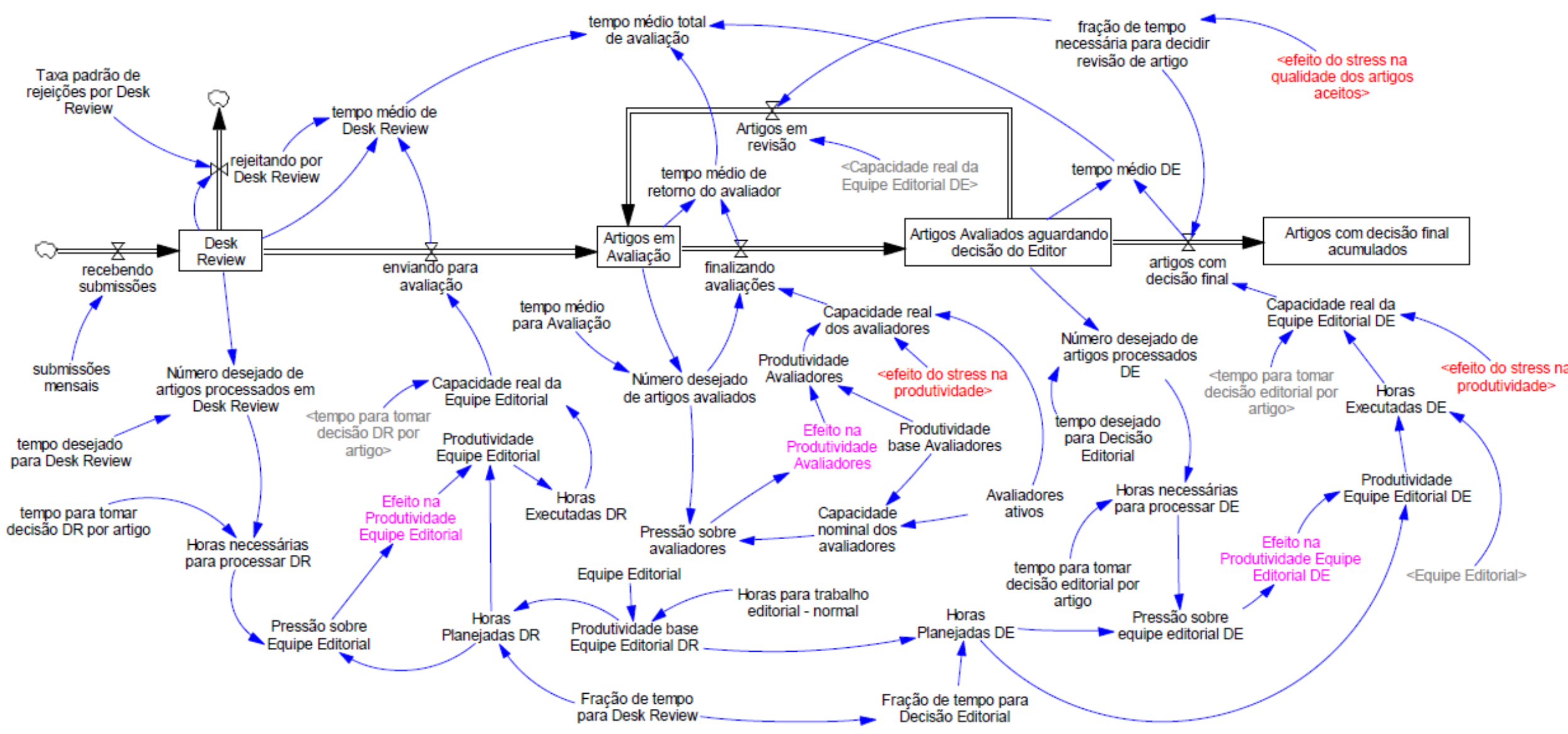

Fonte: Elaboração dos autores 
Figura 6 - Modelo de geração e dissipação de estresse de editores

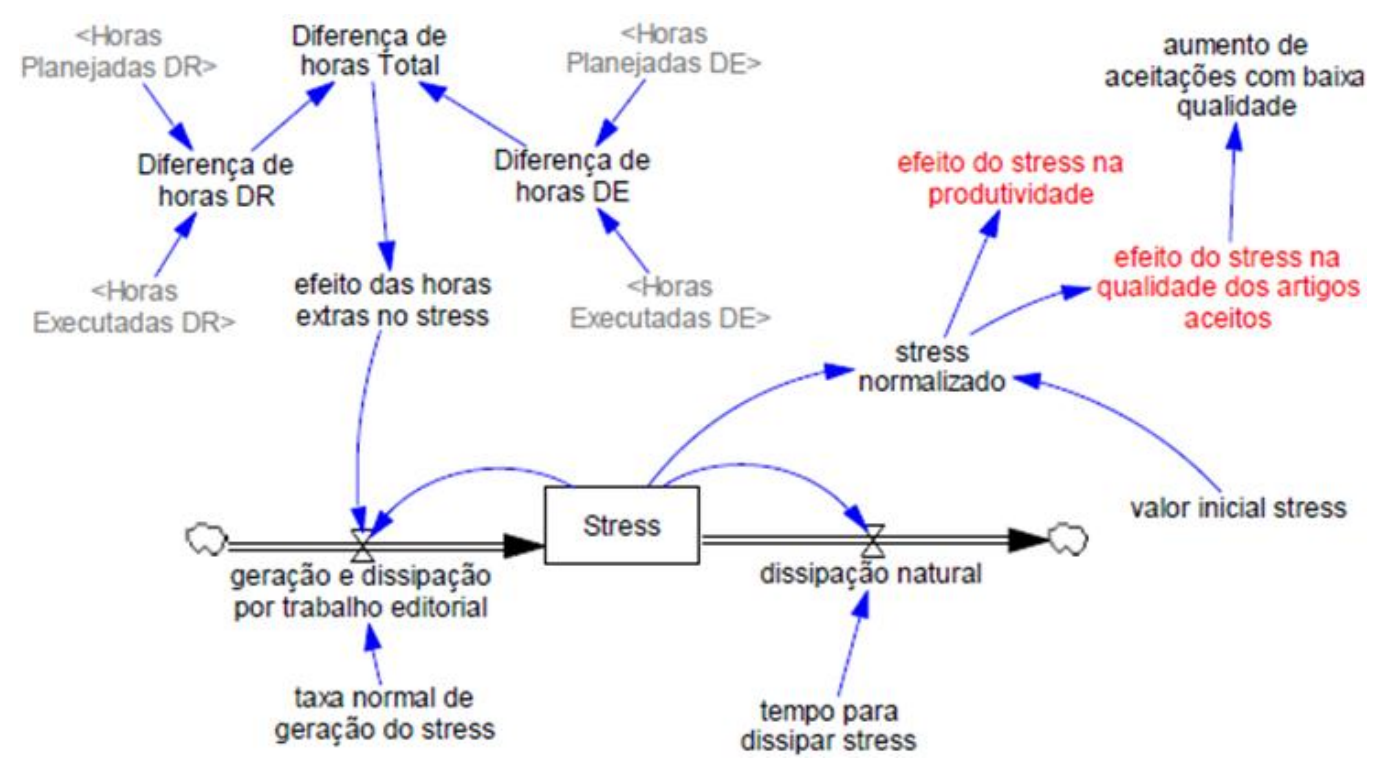

Fonte: Elaboração dos autores a partir de Sant'ana, Uriona-Maldonado e Gontijo (2019).

A sigla DR, nas figuras, refere-se ao esforço dos editores aplicado à desk review ou triagem preliminar de artigos antes da decisão de rejeitar ou enviar para revisão por pares. A sigla DE trata do esforço editorial de processar artigos envolvidos na decisão editorial de aceite ou rejeição, o que inclui a comunicação com revisores, a apreciação dos comentários desses revisores, a decisão e outros arranjos necessários junto aos autores antes da publicação. A Tabela 1 dá o quadro geral dos parâmetros adotados para rodar as simulações, arbitrados ou estimados a partir dos dados coletados nas estatísticas das revistas.

Tabela 1 - Parâmetros iniciais para a simulação de cenários

\begin{tabular}{lcl}
\hline Parâmetro & Valor & Unidade \\
\hline aumento de submissões & 0 & Binário \\
Avaliadores Ativos & 144 & revisores \\
Equipe Editorial & 1 & editor \\
fechando submissões & 0 & Binário \\
Fração de tempo para desk review & 50 & $\%$ \\
"Horas para trabalho editorial - normal” & 40 & horas mensais \\
inicial Artigos aguardando Decisão Editorial & 31 & artigos \\
inicial Artigos em Avaliação & 164 & artigos \\
inicial Desk Review & 34 & artigos \\
mês de início do fechamento de submissões & 24 & número \\
Produtividade base Avaliadores & 0,1 & artigos/revisor/mês
\end{tabular}


redução da taxa de rejeições

redução do step (incremento de submissões)

submissões mensais (vide Figura 3)

switch estresse-qualidade

taxa normal de geração do estresse

tempo desejado para Decisão Editorial

tempo desejado para Desk Review

tempo médio para Avaliação

tempo para dissipar estresse

tempo para tomar decisão DR por artigo

Tempo para tomar decisão editorial por artigo

valor inicial estresse

$\begin{array}{cl}20 & \% \\ 0 & \% \\ 16 & \text { artigos/mês } \\ 1 & \text { Binário } \\ 2,7 & \% \\ 2 & \text { meses } \\ 3 & \text { meses } \\ 4 & \text { meses } \\ 17,6 & \text { meses } \\ 1 & \text { meses } \\ 1,5 & \text { meses } \\ 100 & \text { adimensional }\end{array}$

Fonte: Elaboração dos autores.

As quatro simulações propostas na Figura 2 estendem-se por 72 meses (6 anos), para poder observar mudanças de longo prazo nas variáveis, cujo comportamento é descrito na Figura 7. Conforme o item (c) da figura, em todos os cenários, a simulação inicia com um fluxo mensal de 16 submissões (e 6 rejeições na triagem), com aumento de submissões no mês 12 em 10\% (total 18, cenário A1), $30 \%(21, \mathrm{~A} 2)$ e $50 \%(24, \mathrm{~A} 3)$. 


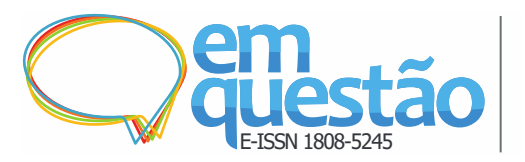

O custo da precariedade: o colapso da saúde dos

editores é também o colapso da revista

Vinícius Medina Kern, Mauricio Uriona-Maldonado

Figura 7 - Comportamento das variáveis nos cenários simulados
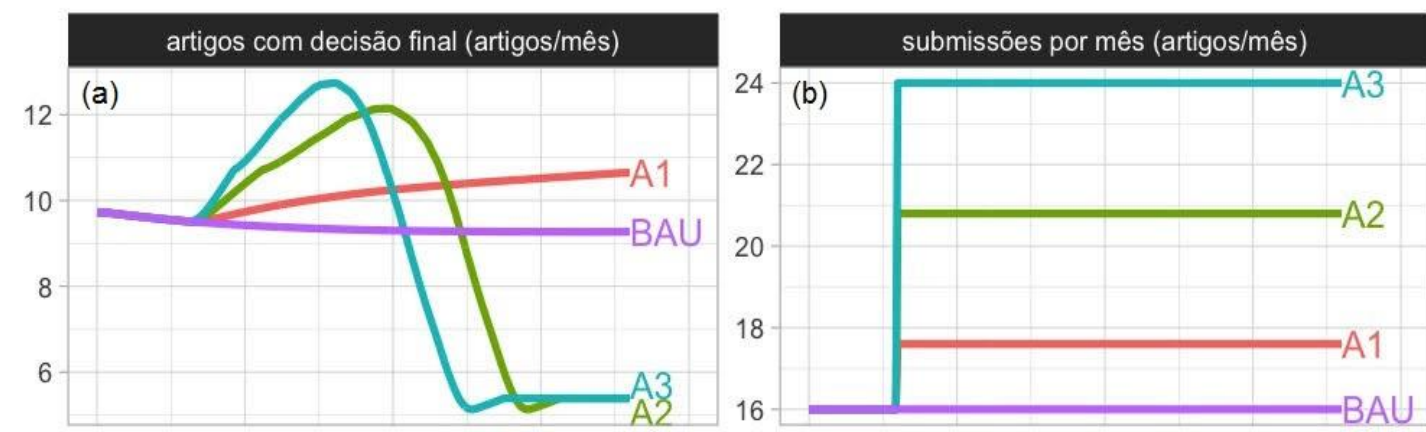

16

capacidade real da equipe editorial DR (artigos/mês)

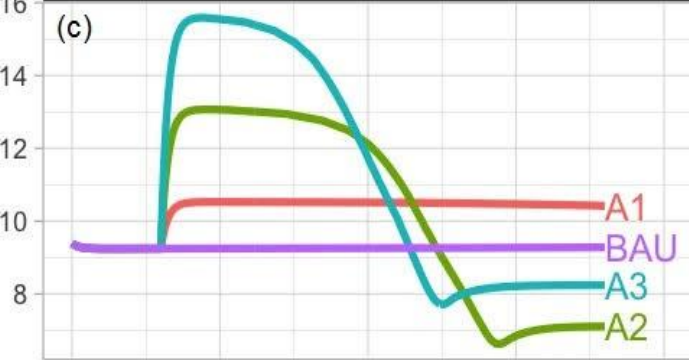

capacidade real da equipe editorial DE (artigos/mês)

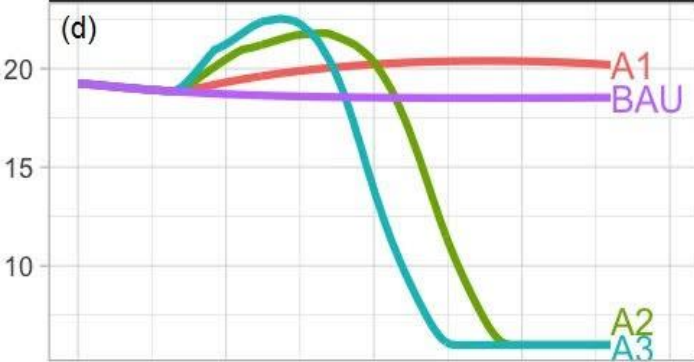

horas planejadas total (horas/mês)
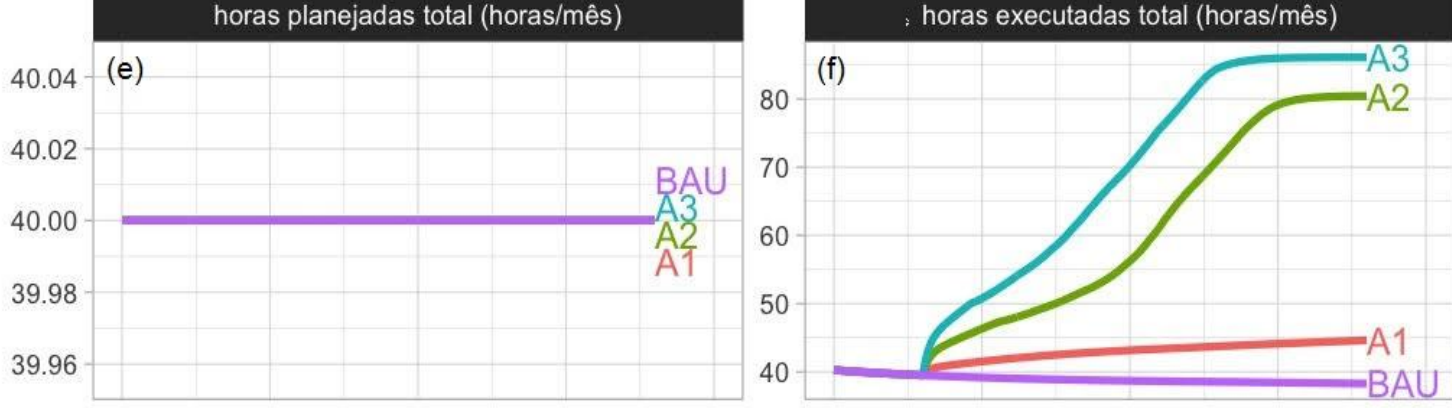

\section{tempo total de avaliação (meses)}
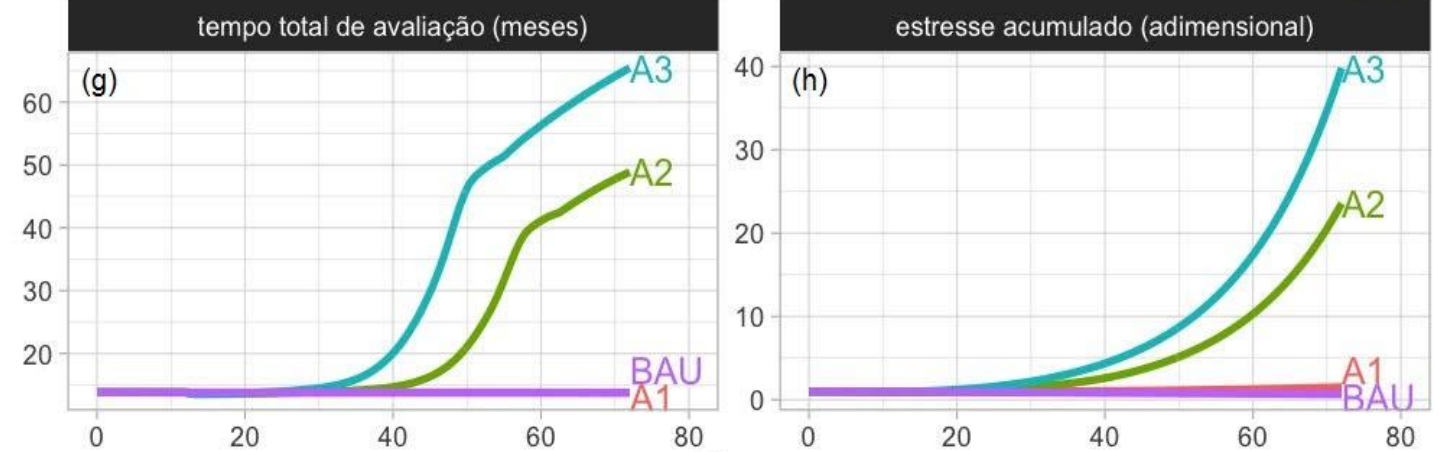

Meses

Fonte: Dados da pesquisa.

No item (a) da Figura 7, os dez artigos (16-6) submetidos e não rejeitados na triagem se mantêm como artigos com decisão final no cenário BAU - ou seja, os editores dão conta de todo o trabalho. No A1, os doze (18-6) artigos mensais sem rejeição na triagem levam a aumento na quantidade de 
decisões pós-triagem, mas esse aumento não se verifica nos cenários A2 e A3 devido ao estresse acumulado dos editores - vide item (h) da figura.

O tempo em meses até a decisão editorial, item (g) na Figura 7, mantémse estável nos cenários BAU e A1, mas aumenta vertiginosamente nos cenários A2 e A3 como resultado do estresse acumulado dos editores, item (h). Os itens (c) e (d) representam o efeito do estresse acumulado em termos de capacidade da equipe editorial para entregar triagens preliminares (desk reviews) - item (c) - e decisões finais - item (d). Os itens (e) e (f) na Figura 7 complementam essa visão do desgaste dos editores: enquanto o item (e) representa a quantidade de horas que se planejava trabalhar, independentemente do cenário, o item (f) mostra o excesso de horas de trabalho editorial à medida que aumenta o fluxo de artigos.

\section{Discussão}

A principal conclusão a partir dos resultados é que o excesso de carga de trabalho pode levar os editores ao esgotamento (burnout) e, como cada revista depende do trabalho de seus editores, o esgotamento desses leva ao colapso da revista. Para evitar o colapso, algumas revistas recorrem a expedientes de efetividade no mínimo discutível. A suspensão ou moratória de submissões é anticientífica, pois exclui a revista do fluxo de produção do conhecimento e força os autores a enviarem seus manuscritos a outro lugar. $\mathrm{O}$ aumento expressivo das rejeições preliminares (desk rejects) pode ser espúrio, mas também pode ser feito justificadamente se os editores examinarem tempestivamente os manuscritos recebidos, com critério científico.

No artigo, prospectamos cenários do fluxo de artigos em avaliação em revistas científicas brasileiras primárias de ciência da informação em acesso aberto sem taxas, bem como do estresse desses editores em resposta a diferentes cargas de trabalho. Dos quatro cenários, dois implicam em estresse excessivo (Figura 7, cenários A2 e A3), com tempo total de avaliação de vários anos e diminuição da capacidade da equipe editorial (como resposta ao estresse excessivo). 
Buscamos simular situações verossímeis, mas partimos de dados empíricos precários, os únicos disponíveis: as estatísticas nos websites das revistas. São de publicação voluntária e, às vezes, estão erradas. Verificamos que algumas revistas alteraram dados históricos de quantidades de artigos enviados para revisão e tempos até decisão ou publicação entre nossas coletas em 12/2017 e em 02/2020. Isso pode decorrer de limitações do software Open Journal System (OJS) - por exemplo, é comum que o primeiro ano em que se divulga quantidade de submissões contenha uma soma de todos os anos anteriores. Talvez por essa precariedade, grande parte das revistas simplesmente não divulga estatísticas.

Propomos que esses cenários são representações verossímeis do parque editorial científico brasileiro em acesso aberto sem taxas. Essa realidade é conhecida informalmente, mas pouco investigada cientificamente. Ainda são poucos os estudos, como o de Oliveira et al. (2020), que reconhecem a complexidade da publicação científica no Brasil, incluindo a precarização do trabalho dos editores de revistas, entre outros aspectos como a tendência à plataformização da ciência, a dependência de infraestruturas de oligopólios, as políticas de avaliação de periódicos científicos e a gestão sem dotação adequada de recursos.

Nossas revistas podem ser enquadradas no pólo "pobre", conforme o levantamento exaustivo das revistas canadenses de Couture (2020): tipicamente sem taxas, mantidas com recursos mínimos, usando websites básicos (com destaque para o OJS). No pólo "rico", websites "profissionais", muitas vezes de sociedades científicas ricas e com taxas de publicação vultosas. No caso brasileiro, várias dessas revistas sem muitos recursos têm entrado em bases de dados de elite como Scopus ou Web of Science, mas sua sobrevivência depende da dedicação de editores sobrecarregados e, em alguns casos, já cometendo atitudes anticientíficas como o fechamento de submissões (para evitar o colapso) ou má conduta, como manter submissões sem qualquer tipo de resposta por meses ou anos.

A sobrecarga aguda é própria de sistemas editoriais precariamente financiados. Revistas com muitos recursos, inclusive as muito prestigiadas, 
estão sujeitas a publicar erro ou fraude, como se pode constatar na base de dados RetractionWatch sobre artigos retratados ou "despublicados" (RETRACTION WATCH, 2021). Revistas em acesso aberto "pay to publish" (pagar para publicar) incluem as "predatórias", que buscam captar taxas sem atenção adequada à qualidade dos artigos e sua avaliação. Dudley (2021) formulou modelos de dinâmica de sistema para estudar revistas em acesso aberto "pay to publish", em particular quanto à barreira econômica para cientistas de países periféricos e ao incentivo às revistas predatórias. Já as revistas estudadas nesta pesquisa, em acesso aberto sem taxas, subfinanciadas, estão sujeitas ao colapso, ou à negligência na avaliação por pares, ou ainda ao fechamento de submissões e a grandes atrasos na avaliação.

Quanto tempo é necessário para avaliar um artigo? Silva e Dobránszki (2017) propõem que o tempo até a decisão editorial não deve ultrapassar cinco a oito meses. Consideram negligência, senão má conduta, a demora excessiva e sem explicações apropriadas aos autores. Nessa perspectiva, os cenários A2 e A3 na Figura 7 são inaceitáveis, embora comuns em algumas revistas da amostra, ainda que essa afirmação parta da experiência pessoal dos autores e seus interlocutores e não das estatísticas publicadas pelas revistas.

No levantamento de Rosa et al. (2020), os tempos medianos são razoáveis e a demora máxima para avaliar artigo chega a 1.229 dias, ou 41 meses, ou quase três anos e meio (em periódico do estrato Qualis A1). Esse caso extremo pode decorrer de impropriedade na conduta editorial, ou não. É um caso isolado (outlier). No entanto, estudos sobre tempos de demora entre submissão e aprovação só podem incluir artigos aceitos e disponíveis para serem pesquisados. Artigos rejeitados não geram estatísticas, exceto contagens brutas. Temos experiência pessoal - e não é difícil encontrar colegas com relatos semelhantes - de artigos rejeitados sem avaliação após anos de espera, com alegações como "não foi possível encontrar revisores" ou "o artigo não se ajusta ao escopo da revista”. Ora, uma decisão de triagem não pode durar anos. Concordamos com Silva e Dobránszki (2017): é negligência, senão má-conduta.

Finalmente, quanta credibilidade é devida a um estudo de simulação como este? Charumilind et al. (2020) lembram que um modelo é tão bom quanto 
seus dados subjacentes (e a qualidade da modelagem-agregamos nós). Pode ajudar a compreender uma situação, mas não é uma "bola de cristal". No manifesto de Saltelli et al. (2020), 22 cientistas afirmam que modelos de simulação devem tratar de questões e não de respostas (e, diríamos nós, de cenários, não de previsões). Recomendam tomar cuidado com os pressupostos do modelo, bem como com a arrogância dos modeladores, com o enquadramento do problema, com as consequências e com o que é desconhecido. Na hierarquia das regras metodológicas gerais de Bunge (1997), as hipóteses, teorias e explicações dinâmicas, que revelam um mecanismo de funcionamento, são superiores a "explicações fenomenológicas", baseadas em aparências, que por sua vez são superiores a modelos de equilíbrio (comuns na economia neoclássica) e meros sumários de dados (identificação de padrões por estatística, como é comum na mineração de dados ou de textos). Nesse sentido, a presente simulação é uma exploração, um estudo preliminar sobre o contexto da sobrecarga de trabalho dos editores e não dispensa a futura pesquisa empírica com dados concretos sobre a saúde e o estresse dos editores.

Afirmamos em trabalho anterior que "[o]s periódicos brasileiros em Ciência da Informação dependem tipicamente de trabalho semivoluntário, com escassa dotação de recursos" (KERN; URIONA-MALDONADO, 2018, p. 258), assumindo que é conhecimento comum. No entanto, os detalhes dessa realidade não são sistematicamente conhecidos; a literatura carece de estudos minuciosos sobre o modelo de negócio de publicação científica em acesso aberto por grupos universitários brasileiros, com financiamento estatal precário.

\section{Financiamento}

O primeiro autor (VMK) é bolsista de produtividade em pesquisa do CNPq.

\section{Agradecimentos}

Os autores agradecem o testemunho de um editor de periódico da área da Saúde sobre seu nível de sobrecarga, na discussão após a apresentação de artigo precursor, no VI Encontro Brasileiro de Bibliometria e Cientometria (KERN; URIONA-MALDONADO, 2018b). 


\section{Referências}

BARNETT, A.; MEWBURN, I.; SCHROTER, S. Working 9 to 5, not the way to make an academic living: observational analysis of manuscript and peer review submissions over time. British Medical Journal, London, v. 367, 2019.

BLATTMANN, U.; SANTOS, R. N. M. Revistas científicas brasileiras e sua visibilidade no acesso aberto. Informação \& Sociedade, João Pessoa, v. 24, n. 3, p. 99-106, 2014.

BUNGE, M. Mechanism and explanation. Philosophy of the Social Sciences, Thousand Oaks, v. 27, n. 4, p. 410-465, 1997.

CAPES. Coordenação de Aperfeiçoamento de Pessoal de Nível Superior. Qualis Periódicos. Plataforma Sucupira, 2018.

CHARUMILIND, S.; EL TURABI, A.; FINN, P.; USHER, O. Demystifying modeling: How quantitative models can - and can't - explain the world. Risk Insights (blog). McKinsey \& Company, 2020.

CORREA, T. P. P.; MIRANDA, A. C. D. A satisfação dos editores das revistas do portal de periódicos da FURG em relação ao sistema eletrônico de editoração de revistas (SEER). Biblios, Pittsburgh, n. 44, p. 41-49, 2011.

COUTURE, M. Canadian OA scholarly journals: An exhaustive survey. Version 1.0. February 6, 2020. (Manuscrito não publicado) Disponível em: https://r-libre.teluq.ca/106/1/MCouture-OA-Canada-report.pdf. Acesso em 14 fev. 2020.

DUDLEY, R. G. The changing landscape of open access publishing: Can open access publishing make the scholarly world more equitable and productive? Journal of Librarianship and Scholarly Communication, Ames, v. 9, n. 1, 2021.

ISEE SYSTEMS. Disponível em: www.iseesystems.com. [2021]. Acesso em: 17 set. 2021.

KERN, V. M.; URIONA-MALDONADO, M. A saúde dos editores de revistas científicas brasileiras de acesso aberto sem taxas: Cenários para um insumo não contabilizado. In: ENCONTRO BRASILEIRO DE BIBLIOMETRIA E CIENTOMETRIA, 7., 2020, Salvador. Anais... Salvador: UFBA, p. 31-37, 2020.

KERN, V. M.; URIONA-MALDONADO, M. Cenários da dinâmica de hipercrescimento e colapso das revistas científicas brasileiras líderes na Ciência da Informação. Em Questão, Porto Alegre, v. 24, ed. esp. 6. EBBC, p. 258-277, 2018. 
KERN, V. M.; URIONA-MALDONADO, M. Hipercrescimento e colapso: Conjeturando cenários da sobrecarga das revistas científicas brasileiras líderes na Ciência da Informação. In: ENCONTRO BRASILEIRO DE BIBLIOMETRIA E CIENTOMETRIA, 6., 2018, Rio de Janeiro. Anais... Rio de Janeiro: UFRJ, p. 90-98, 2018 b.

MEADOWS, D. H.; MEADOWS, D. L.; RANDERS, J.; BEHRENS III, W. W. The limits to growth: a report for The Club of Rome's project on the predicament of mankind. New York: Universe Books, 1972.

MEADOWS, D. H.; RANDERS, J.; MEADOWS, D. L. The limits to growth: the 30-year update. White River Junction: Chelsea Green Publishing, 2004.

MUELLER, S. P. M. O círculo vicioso que prende os periódicos nacionais. DataGramaZero: Revista de Ciência da Informação, Brasília, n. zero, dez. 1999.

OLIVEIRA, T.; GROHMANN, R.; ROSSINI, M. S.; BORGES, G.; FALCÃO, T.; SACRAMENTO, I. Acabou o Quadriênio, e agora? Alguns desafios em relação à avaliação de periódicos na área de comunicação. E-Compós, Brasília, v. 23, p. 1-18, 23 dez. 2020.

RANDERS, J. Elements of the system dynamics method. Portland: Productivity Press, 1980.

RETRACTION WATCH. Retraction Watch database. [2021]. Disponível em: http://retractiondatabase.org. Acesso em: 17 set. 2021.

ROSA, W. P.; GABRIEL JUNIOR, R. F.; MOURA, A. M. M.;

STRASBURGER, K. R.; ALVES, T. M. Análise do tempo de aprovação dos artigos publicados nos Periódicos Brasileiros de Ciência da Informação. In: ENCONTRO BRASILEIRO DE BIBLIOMETRIA E CIENTOMETRIA, 7., 2020, Salvador. Anais... Salvador: UFBA, p. 39-45, 2020.

SALTELLI, A. et al. Five ways to ensure that models serve society: a manifesto (Comment). Nature, Berlim, v. 582, p. 482-484, 2020.

SANT'ANA, J. L. G.; URIONA-MALDONADO, M.; GONTIJO, L. A. Dinâmica de geração e dissipação do estresse na equipe de enfermagem num centro de oncologia. Revista Latino-Americana de Enfermagem, Ribeirão Preto, v. 27, e3156, 2019.

SHINTAKU, M.; SOARES, S. B. C.; BARRAVIERA, B.; LANNES, S. C. S.; RODE, S. M.; FERREIRA JR., R. S. Cenário das revistas geridas pelos participantes do curso de editoração científica da ABEC. Ciência da Informação em Revista, Maceió, v. 5, n. esp., p. 49-57, 2018. 
SILVA, J. A. T.; DOBRÁNSZKI, J. Excessively long editorial decisions and excessively long publication times by journals: causes, risks, consequences, and proposed solutions. Publishing Research Quarterly, New York, v. 33, n. 1, p. 101-108, 2017.

STERMAN, J. D. Business dynamics: systems thinking and modeling for a complex world. Boston: McGraw-Hill, 2000.

STRAUSS, M. Looking back on the Limits of Growth. Smithsonian Magazine, [s.l.], April 2012. Disponível em: https://www.smithsonianmag.com/sciencenature/looking-back-on-the-limits-of-growth-125269840/. Acesso em: 21 set. 2021.

URIONA-MALDONADO, M.; GROBBELAAR, S. S. Innovation system policy analysis through system dynamics modelling: A systematic review. Science and Public Policy, Oxford, v. 46, n. 1, p. 28-44, 2019.

WU, J. T.; LEUNG, K.; LEUNG, G. M. Nowcasting and forecasting the potential domestic and international spread of the 2019-nCoV outbreak originating in Wuhan, China: a modelling study. The Lancet, London, v. 395, n. 10225, p. 689-697, 2020.

\title{
The toll on precariousness: editors' burnout is also the journal's collapse
}

\begin{abstract}
There have been cases of discontinuity, interruption of submissions, and long delays in manuscript evaluation in low-budget Brazilian journals in information science, possibly related to the capacity and health condition of their editors. What is the effect of increased workload on editors' stress? In this article, we simulate four scenarios of the stress of those editors using data on the number of submissions, evaluations, and editorial decisions. We use system dynamics to model stocks and flows of articles through the editorial process, describing four scenarios of the stress of journal editors for 72 months, with different submission loads. In two scenarios, with moderate submission loads, editors keep stress and total evaluation time at acceptable levels, increasing productivity under a small overhead, in accordance with the literature. In the two scenarios with the highest submission overload, the number of hours worked per month escalates, as well as the total evaluation time, and the accumulated stress. The exclusive but insufficient state funding for journals does not provide for high-quality editorial work in a timely manner. This threatens editors' health and favors the interruption of submissions, delays, and even the collapse of those journals.
\end{abstract}


Keywords: Journalology; Open access; System dynamics; Scholarly publishing. Academic productivism; Peer review

Recebido: $26 / 12 / 2020$

Aceito: 05/07/2021

\section{Declaração de autoria}

Concepção e elaboração do estudo: V.M. Kern e M. Uriona-Maldonado

Coleta de dados: V.M. Kern

Elaboração do modelo de dinâmica de sistemas: M. Uriona-Maldonado

Análise e interpretação de dados: V.M. Kern e M. Uriona-Maldonado

Redação: V.M. Kern e M. Uriona-Maldonado

Revisão crítica do manuscrito: V.M. Kern e M. Uriona-Maldonado

\section{Como citar}

KERN, Vinícius Medina; URIONA-MALDONADO, Mauricio. O custo da precariedade: o colapso da saúde dos editores é também o colapso da revista. Em Questão, Porto Alegre, v. 28, n. 1, p. 484-504, 2022. DOI: http://dx.doi.org/10.19132/1808-5245281.484-504

1 Em princípio, essa meta deve estar próxima a zero, considerando que a meta ideal fosse não ter artigos aguardando avaliação. Em outras palavras, essa meta ideal significa que todo artigo que entrasse no processo de avaliação sairia imediatamente dele, já avaliado. Na prática, este valor será sempre diferente de zero. Não é comum as revistas divulgarem, por isso arbitramos um valor - vide seção 3 Resultados. 